\title{
Nursing Children and Young People
}

Article ref: NCYP1216

Date submitted: 27 Mar 2019

Date accepted: 10 July 2019

First/Corresponding Author:

Charlotte Bramanis, Master of Nurse Education

Lecturer in Childrens Nursing

University of West London

College of Nursing

Children have mental health needs too.

Abstract

Children and young people with mental health needs have been long been cared for in specialist facilities to ensure patient safety. Currently, there are not enough beds available for these service users, and children and young people with mental health needs are now being placed onto children's wards in general hospitals. Early intervention has been the key in ensuring children and young people are able to lead a fulfilling life, however with current funding being only $6.4 \%$ of the National Health Services mental health budget, the ability to help at a young age appears unlikely. There is a lack of education provided for healthcare professionals, with many feeling unable to care for these service users the way they need caring for. The creation of clinical practice guidelines for healthcare professionals to follow would increase patient safety, furthermore, implementation of post graduate education on mental health for children's nurses will ensure service users are being cared for with improved patient outcomes. The revised Nursing and Midwifery Council (NMC) standards will ensure newly qualified nurses gain an insight into caring for service users with mental illness before they enter the workforce.

Keywords: Mental health; Young People; Children; Increasing admissions 
Introduction

Children and young people, it is suggested, have more stressors in their lives than ever before in both school and social environments, with the pressure to succeed being immense (Healey, 2017). Mental illness refers to a wide range of mental health conditions that cause a disturbance of feelings and emotions (Healey, 2017). In 2016, in the UK alone, 7.6\% of children over the age of 12 had severe to moderate depression, with $10 \%$ of children over the age of 11 engaging in self-harm (Haefner, 2016). Korkodilos (2016), identifies suicide as being the leading cause of death in young people in the UK over the last five years; with over half of all mental health conditions starting before the age of 14 (Haefner, 2016). These statistics identify the need for change and more opportunity for nurses to understand the complexities of a child or young person with a mental health concern.

Literature suggests addressing mental illness in children and young people when they first present to the General Practice clinic, hospital or outpatients clinic, provides a greater chance of the child or young person learning coping mechanisms to assist them going into adulthood (Royal College of Nursing, 2017; Wollaston, 2014). Early intervention services nationally have become a target for budget cuts, which has resulted in an increased demand for inpatient care as mental illness among children reaches a crisis point (Wollaston, 2014). Extra funding in early interventions would prevent serious problems from developing later in life (Wollaston, 2014). The number of young people with depression between 15 and 16 years of age has doubled between 1980 and 2000 (Hagell, 2013), and 75\% of mental health problems in adult life (excluding dementia) start by the age of 18 (Department of Health, 2015) nevertheless, Child and Adolescent Mental Health Services (CAMHS) currently accounts for just $0.7 \%$ of NHS spending, and around $6.4 \%$ of mental health spending (Young Minds, 2018). Wollaston (2014), discusses the link between an increasing concern for the well-being of service users due to the decrease in availability of beds and nursing healthcare professionals, and the availability of mental health services for young service users; making the current crisis in availability of mental health care (House of Commons 
Health Committee, 2014). The House of Commons Health Committee (2014), attributes the current crisis and prevailing pandemic to the economic crisis, as budgets have been frozen or reduced. Other budgets that have been reduced are for early intervention, resulting in an increased demand for acute services in mental health (Wollaston, 2014).

The United Kingdom has seen a rapid increase in young people with mental illness in the last few years (Haefner, 2016; McDougall, 2016; Thongpriwan et al., 2015), with a political and public interest in access to inpatient beds for these service users peaking. In part this is due to the lack of specialised care, children and young people with mental illness are being transferred to general children's wards rather than specialised facilities (Thomas, 2017). Children and adolescents being cared for in non-suitable environments dates back to the beginning of the millennium (Buckley, 2010; Greening \& Hunt, 2017; McDougall, 2016; Thomas, 2017), however, it is increasing to a point that there is often at least one mental health service user on the ward at all times (McDougall, 2016). Childrens nurses often have little or no mental health training (Thomas, 2017), which previously was a contributing factor to why $82 \%$ of nurses refuse to be allocated or accountable for patients with mental illness (Reeves, 2011). In 2014, the Guardian reported on 'whistle-blower' nurses (Anonymous, 2014), who stated they felt ill equipped to care for patients with mental health problems and made a call for the government to make changes to the current provision of services. These concerns were also raised in the Shape of Caring Review (Willis, 2015), where recommendations were made that all nurses should have knowledge of illnesses across the lifespan. The Nursing and Midwifery Council (NMC) have changed the standards of proficiency for nurses (NMC, 2018) to address concerns raised by the Shape of Caring Review (Willis, 2015), and now incorporates nurses having an understanding of the best practice approaches to mental health and well-being, and to address basic mental health first aid (NMC, 2018). The changes in the NMC standards means universities will need to change the curriculum, allowing students to graduate with a basic understanding of mental health and early intervention. The National Health Service (NHS), recognises the mental 
health pandemic currently affecting hospitals and are working with Hospital Trusts to rectify deficits and create better facilities for admitted mental health patients (Greening \& Hunt, 2017), NHS healthcare workers need to pool their resources and enable cross field education; the transference of knowledge from the mental health team to the children's team ensuring children and young people are cared for more suitably (Greening \& Hunt, 2017; Wollaston, 2014).

The number of children and young people requiring admission for treatment of an acute episode of mental illness has increased by $68 \%$ over the last ten years; this increase leading to a greater demand on the NHS for the vital inpatient and outpatient services, ultimately resulting in extended waiting times (House of Commons Health Committee, 2014). Untreated mental issues during childhood have been attributed to a lifetime of aggression, within an increase in violence, crime, and unemployment as well as other risk-taking behaviours (Joint Commissioning Panel for Mental Health, 2015). The reported reduction in bed capacity for inpatients has further attributed to children's emergency departments seeing a higher presentation of children and young people with complications secondary to poor mental health, including an increase of drug use and dependency (Joint Commissioning Panel for Mental Health, 2015). By increasing the knowledge of healthcare professionals in mental illness, there would be a decrease in service users requiring mental health care in adulthood, reducing the pressure on the NHS further.

The Joint Commissioning Panel for Mental Health (2015) postulated all healthcare professionals should be educated in mental health conditions; children's nurses have not been given the appropriate education and training to care for children and young people with mental illness (Bolland, Richardson, \& Calnan, 2017; McDougall, 2016; Reeves, 2011; Thomas, 2017). Thomas (2017), conducted a retrospective study of third year students, which identified a lack of mental health education for children's nurses. Thongpriwan et al. (2015), propose healthcare professionals are more willing to expand their knowledge of mental health at post graduate level when they are given a basic understanding at an 
undergraduate level; suggesting post graduate nurses feel less apprehensive when they have prior knowledge. Bingham (2018), identifies health professionals can appear to discriminate or have stigmatising views towards people with mental health illness, and these can be sub-conscious. A study of these biases validated the incorporation of a guided clinical experience in an acute mental health unit during the first year of undergraduate studies to limit stigmatising beliefs (Bingham, 2018). The incorporation of mental health into the new NMC standards for nurse training is a clear step towards ensuring healthcare professionals understand the needs of patients with a mental health concern. The Shape of Caring Report (Willis, 2015), proposes increasing nurses knowledge of mental illness across all fields to permit a more active engagement and understanding of patients' needs, and all fields of nurses developing an understanding of mental healthcare issues. A clinical practice guideline (CPG) in conjunction with clinical care groups (CCG), would ensure the best outcome for each patient.

CPG's are widespread and have been developing and improving patient outcomes for over forty years (Murad, 2017). CPG's are a peer-reviewed approach to caring for patients, based on literature reviews to ensure evidence-based practice (American Academy of Pediatrics, 2015). CPG's must comply to NHS standards and be reviewed by a rigorous selection panel to ensure they meet the NMC code before implementation in the workplace (National Institute for Health and Care Excellence, 2012). CPG's are imperative to enable evidencebased practice and gold standard care (American Academy of Pediatrics, 2015), however, throughout the NHS, there are many children's wards without a CPG to give guidance to healthcare professionals in relation to mental health admissions (NHS England, 2019). Specialised CAHMS units have specific CPG's, and where these guidelines are used, positive effects on children and young people were evident (Royal College of Psychiatrists, 2015). NHS England (2019), are developing CCG's in response to the Future in Mind proposal (Department of Health, 2015), and are now beginning to engrain CCG's into CAMHS inpatient services (NHS England, 2019), however this is a Monday to Friday service 
to support children and young people with mental illness. Burton, Pavord, \& Williams (2014), identify that children and young people are often admitted to the ward overnight, during which time there is no access to specialist treatment or CCG's, and the service users are often placed in an open bay with up to five other children or young people nearby. Patient safety is paramount in a hospital environment (McDougall, 2016), current research identifies the lack of funding for specialist mental health units being detrimental to the child or young person's future as they are not receiving the care they deserve (Bolland et al., 2017; Buckley, 2010; Greening \& Hunt, 2017; Thomas, 2017; Wollaston, 2014). If a CPG were in place for overnight admissions there would be an increase in service user safety as an initial care plan would be created, avoiding the risk of these service users potentially having missed referrals due to oversights. A mental health specific CPG would ensure that gold standard care is given to each service user on admission and throughout their stay on the ward leading through to discharge into a stable and safe environment (National Institute for Health and Care Excellence, 2012). To assist in maintaining a gold standard of care the need for in-house training from the CAHMS team for the nurses in the children's ward is apparent (Bolland et al., 2017; Greening \& Hunt, 2017; McDougall, 2016; Thomas, 2017; Thongpriwan et al., 2015; Wollaston, 2014). With the increase of young metal health service users attending the ward at an all-time high (Thongpriwan et al., 2015), it is imperative that hospitals begin educating post graduate children's nurses as soon as possible to support and meet the needs of service users.

To enable a well-developed teaching plan and learning outcomes for post graduate nurses, nurse educators from mental health and children's nursing will need to work alongside each other to ensure healthcare professionals are equipped with the right level of knowledge to increase staff engagement (Roussel et al., 2015). Nurse educators should be constantly striving to develop competencies to ensure the best evidence-based practice for service users (Holloway, Arcus, \& Orsborn, 2018), furthermore, to engage the workforce and enhance retention as nurses are equipped to develop skills and competencies. Govranos 
and Newton (2014), suggest nurses desire ongoing development and postulate that a ward environment is the best place to achieve this with education at a ward-based level enabling healthcare professionals to feel empowered in the care of patients with mental illnesses. To ensure any education plan for ongoing development is sustainable it is recommended the ward regularly review the training needs of the ward staff to ensure nurses are receiving relevant and up-to-date knowledge (Holloway et al., 2018). Champion models have been developed for many years and are widely used in the NHS (Sutton et al., 2018). An adolescent champion model was implemented in a UK hospital (Riley, Patterson, Lane, Won, and Ranalli, 2018), and analysis of the model was from the perspective of the patient; identifying that patient feedback was positive and there was an increase in patient satisfaction with the use of the model. It has been so successful that the role is now an integral part of the workforce. The idea of a champion in the workplace is becoming ever increasingly popular as evidence-based practice is leading the changes (Luz et al., 2018; Riley et al., 2018). The creation of an adolescent champion throughout the NHS for children and young people with mental illness would permit an individual point of contact for mental health education or service user concerns, making healthcare professionals feel more comfortable asking questions. The adolescent mental health champion should work in collaboration with the mental health team and CCG to ensure evidence-based practice.

\section{Conclusion}

The need for an urgent change in care of children and young people has been identified and there is clear evidence to suggest this needs to be implemented soon. Although there are CCG's for inpatients, there is a lack of standards for admissions outside if Monday to Friday. The creation of CPG's nationwide would assist in the care of these service users. The change in NMC standards of proficiency will enable all newly qualified nurses from September 2020 to have a basic understanding of mental illness and best practice. These changes alongside mental health training for all qualified nurses will ensure children and young people receive evidence based care when admitted to a general children's ward. 
References

American Academy of Pediatrics. (2015). Pediatric clinical practice guidelines \& policies: a compendium of evidence-based research for pediatric practice (15th ed.). Elk Grove Village, IL: American Academy of Pediatrics.

Anonymous. (2014). As a nurse I don't feel equipped to treat patients with mental health problems. Retrieved from https://www.theguardian.com/healthcare-network/views-from-thenhs-frontline/2014/sep/29/not-equipped-treat-patients-mental-health-problems

Bingham, H. (2018). Educational intervention to decrease stigmatizing attitudes of undergraduate nurses towards people with mental illness. International Journal of Mental Health Nursing. 27(1). 480-491. doi: 10.1111/inm.12322

Bolland, R., Richardson, J., \& Calnan, R. (2017). How professionals should communicate with children who have mental healthcare needs. Nursing Children and Young People 29(1), 20. doi: $10.7748 /$ ncyp.2017.e814

Buckley, S. (2010). Caring for those with mental health conditions on a children's ward. British Journal of Nursing, 19(19), 1226. doi: 10.7748/ncyp.2017.e814

Burton, M., Pavord, E., \& Williams, B. (2014). An introduction to child and adolescent mental health. London: Sage.

Department of Health (2015). Future in mind. NHS England. Retrieved from https://webarchive.nationalarchives.gov.uk/20170422110702/https://www.gov.uk/governmen t/uploads/system/uploads/attachment_data/file/414024/Childrens_Mental_Health.pdf

Govranos, M., \& Newton, J. (2014) Exploring ward nurses' perceptions of continuing education in clinical settings. Nurse Education Today. 34(4), 655-660. doi:10.1016/j.nedt.2013.07.003

Greening, J., \& Hunt, J. (2017). Transforming Children and Young People's Mental Health Provision: a Green Paper. London: APS Group on behalf of the Controller of Her Majesty's. Retrieved from https://assets.publishing.service.gov.uk/government/uploads/system/uploads/attachment_da ta/file/664855/Transforming_children_and_young_people_s_mental_health_provision.pdf Haefner, J. (2016). Primary care management of depression in children and adolescents. The Nurse practitioner, 41(6), 38-45. doi: 10.1097/01.NPR.0000483046.97563.4c 7 Hagell, A. (2013). Social trends and mental health: introducing the main findings. London: Nuffield Foundation. 
Hamilton, S. (2013). Assessing knowledge skills in the NHS: a training needs analysis approach. Health Information \& Libraries Journal, 30(2), 168-173. doi: 10.1111/hir.12023 Healey, J. (2017). Youth Mental Health. Thirroul, NSW: Spinney Press.

Holloway, K., Arcus, K., \& Orsborn, G. (2018). Training needs analysis - The essential first step for continuing professional development design. Nurse Education in Practice, 28, 7-12. doi: 10.1016/j.nepr.2017.09.001

House of Commons Health Committee. (2014). Children's and adolescents' mental health and CAMHS. London: The Stationary Office. Retrieved from https://publications.parliament.uk/pa/cm201415/cmselect/cmhealth/342/342.pdf Joint Commissioning Panel for Mental Health. (2015). Guidance for commissioners of mental health services for young people making the transition from child and adolescent to adult services. Raffertys. Retrieved from www.jcpmh.info

Korkodilos, M. (2016). Reducing child mortality in London. London: Retrieved from https://www.gov.uk/government/publications

Luz, S., Shadmi, E., Admi, H., Peterfreund, I., \& Drach-Zahavy, A. (2018). Characteristics and behaviours of formal versus informal nurse champions and their relationship to innovation success. Journal of Advanced Nursing. 75(1). doi: 10.1111/jan.13838

McDougall, T. (2016). Child and adolescent mental health inpatient nursing: A call for action. British Journal of Mental Health Nursing, 5(1), 10-14. doi: 10.12968/bjmh.2016.5.1.10 Murad, M. (2017). Clinical practice guidelines: a primer on development and dissemination. Mayo Clinic Proceedings. 92(3), 423-434. doi: 10.1016/j.mayocp.2017.01.001

National Institute for Health and Care Excellence. (2012). The guidelines manual. London: NICE. Retrieved from https://www.nice.org.uk/process/pmg6/chapter/writing-the-clinicalguideline-and-the-role-of-the-nice-editors

NHS England (2019). Children and Adolescent Mental Health Service (CAMHs) Inpatient Services. Retrieved from https://www.england.nhs.uk/mental-health/cyp/children-andadolescent-mental-health-service-inpatient-services/

Nursing and Midwifery Council (2018). Standards of proficiency for nurses. Available at https://www.nmc.org.uk/standards/standards-for-nurses

Reeves, A. (2011). An analysis of the training needs of frontline staff in inpatient CAMHS. Retrieved from www.qualityinevidence.com 
Riley, M., Patterson, V., Lane, J. C., Won, K., \& Ranalli, L. (2018). The Adolescent Champion Model: Primary Care Becomes Adolescent-Centered via Targeted Quality Improvement. The Journal of Pediatrics. 193, 229-236. doi: 10.1016/j.jpeds.2017.09.084

Roussel, L., Harris, J., \& Thomas, P. (2015). Management and leadership for nurse administrators (7th ed.). Sudbury: Jones and Bartlett.

Royal College of Nursing. (2017). Child and Adolescent Mental Health. London: RCN. Royal College of Psychiatrists (2015) CG28 Depression in children and young people: Full guideline. Retrieved from https://www.nice.org.uk/guidance/cg28/evidence/full-guideline-pdf193488883

Sutton, S., Marston, L., Street, A., Masood, S., Edmondson, C., \& Westwood, A. (2018). Involving young people in their care: The Walsall school nurse champions. British Journal of School Nursing. 13(3). doi: 10.12968/bjsn.2018.13.3.136

Thomas, L. (2017). Nursing children and young people: what mental health training is required? British Journal of Nursing. 26(4), 234. doi: 10.12968/bjon.2017.26.4.234

Thongpriwan, V., Leuck, S., Powell, R., Young, S., Schuler, S., \& Hughes, R. (2015). Undergraduate nursing students' attitudes toward mental health nursing. Nurse Education Today. 35(8), 948. doi: 10.1016/j.nedt.2015.03.011

Willis, G. (2015). Shape of Caring: A review of the future education and training of registered nurses and care assistants. United Kingdom: Nursing and Midwifery Council.Retrieved from https://www.hee.nhs.uk/sites/default/files/documents/2348-Shape-of-caring-reviewFINAL.pdf

Wollaston, S. (2014). CAMHS has 'serious and deeply ingrained problems'. Nursing Standard, 29(11), 8. doi: 10.7748/ns.29.11.8.s6

Young Minds (2018). Children's mental health funding not going where it should be. Retrieved from https://youngminds.org.uk/about-us/media-centre/press-releases/children-smental-health-funding-not-going-where-it-should-be/ 